\title{
Sarcopenia is associated with severe erectile dysfunction in older adults: a population-based cohort study
}

\author{
Hyungchul Park ${ }^{1}$, Il-Young Jang ${ }^{1,2}$, Minkyu Han ${ }^{3}$, Heayon Lee ${ }^{1}$, Hee-Won Jung ${ }^{4}$, Eunju Lee ${ }^{1}$, and \\ Dae Hyun Kim ${ }^{5,6}$
}

\begin{abstract}
${ }^{1}$ Department of Internal Medicine, Asan Medical Center, University of Ulsan College of Medicine, Seoul; ${ }^{2}$ Pyeongchang Health Center \& Country Hospital, Pyeongchang; ${ }^{3}$ Department of Clinical Epidemiology and Biostatistics, Asan Medical Center, University of Ulsan College of Medicine, Seoul; ${ }^{4}$ Division of Geriatrics, Department of Internal Medicine, Seoul National University Bundang Hospital, Seongnam, Korea; ${ }^{5} \mathrm{Hinda}$ and Arthur Marcus Institute for Aging Research, Hebrew Senior Life, Boston, MA; ${ }^{6}$ Division of Gerontology, Department of Medicine, Beth Israel Deaconess Medical Center, Boston, MA, USA
\end{abstract}

Received: May 2, 2019

Revised : July 8, 2019

Accepted: July 22, 2019

Correspondence to

Eunju Lee, M.D.

Department of Internal

Medicine, Asan Medical Center,

University of Ulsan College of

Medicine, 88 Olympic-ro 43-gil,

Songpa-gu, Seoul 05505, Korea

Tel: +82-2-3010-3308

Fax: +82-2-476-0824

E-mail: eunjulee@amc.seoul.kr

https://orcid.org/0000-0003-

4612-0625
Background/Aims: Sarcopenia and erectile dysfunction (ED) are associated with poor health and quality of life in older men. We investigate the association between sarcopenia and severe ED in community-dwelling older men.

Methods: We prospectively assessed sarcopenia and ED in 519, community-dwelling, older men (mean age, 74.0) in Pyeongchang, Korea, in 2016 to 2017. Sarcopenia was based on muscle mass, grip strength, and gait speed according to the Asian Working Group consensus algorithm. Severe ED was defined as 5-item International Index of Erectile Function questionnaire score under 8. Logistic regressions were used to study associations between incident severe ED and sarcopenia, after adjusting age, cardiovascular risk factors, depression, and polypharmacy.

Results: The prevalence of severe ED was $52.4 \%$ and that of sarcopenia was $31.6 \%$. At baseline, the prevalence of severe ED was higher in men with sarcopenia than in those without (73.2\% vs. $42.8 \%$; adjusted odds ratio [aOR], $1.89 ; 95 \%$ confidence interval [CI], 1.18 to $3.03 ; p=0.008$ ). Slow gait speed (aOR, 2.80; 95\% CI, 1.18 to 6.62; $p=0.019)$ and decreased muscle mass (aOR, 2.54; $95 \% \mathrm{CI}, 1.11$ to $5.81 ; p=0.027)$ were associated with the incidence of severe ED, while decreased grip strength (aOR, $0.76 ; 95 \%$ CI, 0.30 to $1.91 ; p=0.564$ ) was not.

Conclusions: Sarcopenia was associated with severe ED. Slow gait speed, and decreased muscle mass was independently associated with incident severe ED at 1 year. Further research is warranted to examine whether an intervention targeting these components can prevent severe ED.

Keywords: Erectile dysfunction; Geriatric assessment; Longitudinal study; Public health practice; Sarcopenia

\section{INTRODUCTION}

Erectile dysfunction (ED) is defined as a consistent or repetitive inability to obtain or maintain a penile erection of sufficient rigidity and duration for sexual intercourse [1]. ED is highly prevalent worldwide, currently affecting 150 million men and 322 million by the year 2025 [2]. Approximately half of men over 70 years of age have 
ED with moderate to severe symptoms $[3,4]$ and thus experience poor quality of life [5]. Sarcopenia, which is characterized by decreased muscle mass, strength and/ or physical performance, is an important risk factor for an adverse health outcome and poor quality of life [6-9].

Except for the mechanism of direct damage to the nerves associated with erection, various causes, including vascular, psychogenic, and hormonal factors, are associated with ED. Among the vascular factors, cardiovascular disease (CVD), hypertension, diabetes mellitus, dyslipidemia, and smoking are well-known risk factors for ED $[3,4,10]$. Hormonal and psychogenic factors are represented by decreased androgen hormone level and depression [1].

Androgen hormone was known to be one of the causes of ED and sarcopenia. Although the diagnosis of ED and low androgen hormone could be uncertain, erections are androgen-dependent, and androgen replacement therapy for a patient with a low bioavailable androgen level has been confirmed $[1,11,12]$. Sarcopenia can be associated with low androgen levels because low levels of androgen are associated with decreased anabolism of skeletal muscle. Also, as a treatment for sarcopenia, supplementation of androgen hormone has been suggested $[13,14]$. However, it is not clear whether sarcopenia is an independent risk factor for ED. Only the association between ED and sarcopenia in men with diabetes has been reported in a previous study [15].

Therefore, the aim of this study is to analyze the relationship between sarcopenia and ED in a community setting. We prospectively assessed sarcopenia and ED over a 1-year period in order to investigate the association of sarcopenia and its components with prevalent and incident ED, excluding the established risk factors for ED in community-dwelling older men.

\section{METHODS}

\section{Study population and design}

The population of this study was analyzed from the Aging Study of Pyeongchang Rural Area (ASPRA), a population-based, prospective cohort study of frailty and geriatric syndromes that has covered 1,650 community-dwelling, older adults since December 2014 [16]. The detailed design and method of ASPRA have been de- scribed elsewhere $[16,17]$. Briefly, older adults residing in Pyeongchang County, Gangwon Province, Korea, located $180 \mathrm{~km}$ east of Seoul, were enrolled through public healthcare networks. Comprehensive geriatric assessments were performed annually. The inclusion criteria for participation in ASPRA were (1) age $\geq 65$ years, (2) registered in the National Healthcare Services, (3) ambulatory with or without an assistive device, (4) living at home, and (5) able to provide informed consent. The exclusion criteria were (1) living in a nursing home, (2) hospitalized, or (3) bed-ridden and receiving nursing-home-level care at the time of their enrollment. We have shown that the ASPRA cohort included over $90 \%$ of the eligible population in the study area, and the characteristics of the participants were comparable to those of a nationally representative sample of the Korean rural population, except for a higher proportion with agriculture as an occupation and a lower proportion with a formal education [16].

Of the total of 524 men who participated in the ASPRA cohort, as five were excluded due to incomplete physical function assessment, we analyzed the data from 519 (99.0\%) men who completed assessments for ED between January 1, 2016 and December 31, 2017 for the present study. Of these 519 men, 272 with severe ED were evaluated at baseline. Those without severe ED at baseline $(n=247)$ were prospectively analyzed. After 1 year, 50 men were excluded due to follow-up loss, i.e., 21 who declined follow-up participation, 17 follow-up loss, eight who moved to another county, three who were institutionalized, and one who died. Therefore, the remaining 197 men were evaluated (Fig. 1). The protocol of this study was approved by the Institutional Review Board of Asan Medical Center, Seoul, Korea (IRB No. 2015-0673). Written informed consent was obtained from all participants.

\section{Measurement of erectile dysfunction}

The five-item version of the International Index of Erectile Function (IIEF) was used to evaluate sexual desire and ED with a scoring range of 5 to 25 [18]. Male physicians administered the IIEF-5 questionnaire to participants in a private area. Based on the IIEF-5 score, participants were classified as having severe $\operatorname{ED}(5$ to 7$)$, moderate ED (8 to 11), mild to moderate ED (12 to 16), mild ED (17 to 21), or no ED (22 to 25) [18]. The outcome of interest was severe ED because severity of erectile dys- 


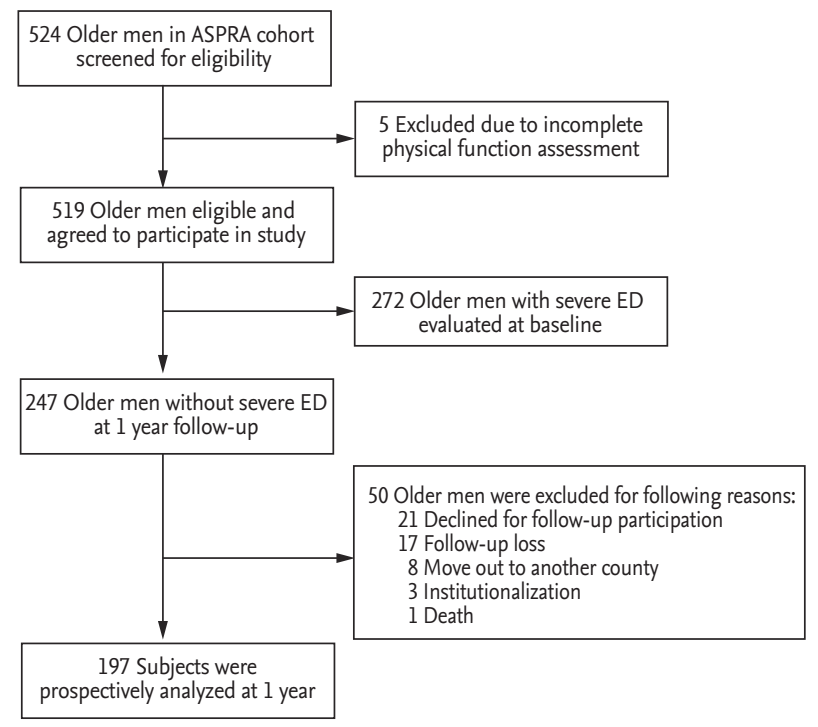

Figure 1. Recruitment of the study population. ASPRA, Aging Study of PyeongChang Rural Area; ED, erectile dysfunction.

function is associated with poorer life quality [5] and a higher prevalence of frailty was observed in men with severe ED [19].

\section{Measurement of sarcopenia}

Nurses with specific, related training conducted measurements of muscle mass, grip strength, and gait speed to assess sarcopenia.

\section{Muscle mass}

Bioelectrical impedance analysis (InBody 620; InBody, Seoul, Korea) with measuring frequencies of 5,50 , and $500 \mathrm{kHz}$ was used to evaluate body composition including the total mass and the lean mass. Bioelectrical impedance analysis was previously validated against dual-energy X-ray absorptiometry [20]. All of the patients were asked to fast overnight and four-limb impedance was measured by the device with the patient in a standing position. Appendicular skeletal muscle mass (ASM) was calculated as the sum of the lean mass of both arms and legs provided by the manufacturer's algorithm. ASM was divided by the height squared (ASM/ht ${ }^{2}$ ) in order to allow comparison of the muscle mass between the participants.

\section{Grip strength}

A spring-based dynamometer (T.K.K. 5401 Grip-D;
Takei, Tokyo, Japan) was used to assess the handgrip strength (kg) in both arms [21]. Participants were asked to hold the dynamometer as strongly as possible while in a comfortably sitting position with their arm bent $90^{\circ}$ over the knee [22]. All tests were conducted twice at intervals more than one minute. The maximum value from the dominant arm was used for the analysis.

\section{Gait speed}

In order to evaluate the usual gait speed, participants were instructed to walk a total of $7 \mathrm{~m}$ on a level indoor surface and at the pace most comfortable and usual for them. A trained tester measured the $4 \mathrm{~m}$ distance transit time using a digital stopwatch between the first foot step at the starting line and the first footstep at the $4 \mathrm{~m}$ line. The $1.5 \mathrm{~m}$ intervals of each acceleration and decelerating section were excluded from the measurement [8]. The result was reported according to the gait speed $(\mathrm{m} / \mathrm{sec})[23]$.

\section{Definition of sarcopenia}

We determined sarcopenia according to the Asian Working Group for Sarcopenia consensus algorithm. Briefly, among older adults with low handgrip strength and/or slow gait speed and who has low muscle mass at the same time was classified as having sarcopenia [8]. Decreased muscle mass was defined as the sex-specific lowest quintile of ASM/ $\mathrm{ht}^{2}[8,24]$, which has been widely adapted in sarcopenia definitions, and the clinical relevance of this measure in the ASPRA population has previously been reported [25]. Decreased grip strength was defined as handgrip strength less than $26 \mathrm{~kg}$ for men and less than $18 \mathrm{~kg}$ for women. Slow gait speed was defined as gait speed less than $0.8 \mathrm{~m} / \mathrm{sec}$ [8].

\section{Other measurements}

Other geriatric conditions were assessed by trained nurses using standardized instruments. Disability was defined as requiring assistance in performing any of the seven activities of daily living (ADL), i.e., ADL, bathing, continence, dressing, eating, toileting, transferring, and washing face and hands, or the 10 instrumental activities of daily living (IADL), i.e., IADL, food preparation, household chores, going out a short distance, grooming, handling finances, laundry, managing their own medications, shopping, transportation, and using a telephone [26]. Cognitive dysfunction was determined if the 
Table 1. Baseline characteristics between sarcopenia status $(n=519)$

\begin{tabular}{|c|c|c|c|}
\hline Characteristic & Sarcopenia $(-)(\mathrm{n}=355)$ & Sarcopenia $(+)(n=164)$ & $p$ value \\
\hline Age & $72.4 \pm 5.05$ & $77 \cdot 3 \pm 5.87$ & $<0.001$ \\
\hline Smoking & $52(14.7)$ & $35(21.3)$ & 0.076 \\
\hline CVD & $216(60.9)$ & $88(53.7)$ & 0.147 \\
\hline Diabetes & $64(18.0)$ & $32(19 \cdot 5)$ & 0.777 \\
\hline Depression & $5(1.4)$ & $7(4 \cdot 3)$ & $0.05^{8}$ \\
\hline Polypharmacy & $60(16.9)$ & $39(23.8)$ & 0.083 \\
\hline Cognitive dysfunction & $28(7 \cdot 9)$ & $58(35 \cdot 4)$ & $<0.001$ \\
\hline Fall in past year & $45(12.7)$ & $26(15 \cdot 9)$ & 0.327 \\
\hline At risk of malnutrition & $73(20.6)$ & $92(56.1)$ & $<0.001$ \\
\hline ADL disability & $23(6.5)$ & $24(14.6)$ & 0.003 \\
\hline IADL disability & $35(9 \cdot 9)$ & $42(25 \cdot 6)$ & $<0.001$ \\
\hline Decreased muscle mass $^{\mathrm{a}}$ & $17(4.8)$ & $164(100)$ & $<0.001$ \\
\hline Slow gait speed ${ }^{b}$ & $241(67.9)$ & $153(93 \cdot 3)$ & $<0.001$ \\
\hline Decreased grip strength $^{c}$ & $55(15 \cdot 5)$ & $90(54.9)$ & $<0.001$ \\
\hline
\end{tabular}

Values are presented as mean \pm SD or number (\%).

CVD, cardiovascular disease; ADL, activities of daily living; IADL, instrumental activities of daily living.

${ }^{a}$ Decreased muscle mass, the sex-specific lowest quintile of appendicular skeletal mass/height ${ }^{2}$.

${ }^{\mathrm{b}}$ Slow gait speed, gait speed $<0.8 \mathrm{~m} / \mathrm{sec}$.

${ }^{\mathrm{c}}$ Decreased grip strength, grip strength $<26 \mathrm{~kg}$.

score on the Korean version of the Mini-Mental State Examination was $<24$ [27]. Depression was determined if the score on the Korean version of the Center for Epidemiological Studies Depression scale was $\geq 21$ [28]. A risk for malnutrition was defined as a Mini-Nutritional Assessment-Short Form score of $\leq 11$ points [29]. Underlying comorbidities, i.e., hypertension, diabetes mellitus, CVD, and dyslipidemia, a history of falling down during the past year, and the amount of cigarette smoking were self-reported by participants. CVD was defined as a participant with any of the following: hypertension; any heart disease, e.g., ischemic heart disease, heart failure, a history of stroke, and dyslipidemia.

\section{Statistical analysis}

Baseline characteristics were compared between the participants with and those without sarcopenia, using a two-sample $t$ test for continuous variables and a chisquare test for categorical variables. We examined the prevalence of ED in those with and those without sarcopenia at baseline. Logistic regression was used to estimate the odds ratios (ORs) and 95\% confidence intervals
(CIs) for severe ED associated with sarcopenia and the three sarcopenia components, after adjusting for patient age, smoking, CVD, diabetes, depression, and polypharmacy. Among those without severe ED at baseline, we examined the incidence of severe ED in those with and those without sarcopenia and its components. The ORs and $95 \%$ CIs for incident-severe ED associated with sarcopenia and its components were estimated using logistic regression to adjust for the above-mentioned variables. Statistical analysis was performed using SPSS version 18.0 (IBM Corporation, Armonk, NY, USA). A two-sided $p$ value $<0.05$ was considered statistically significant.

\section{RESULTS}

\section{Characteristics of study participants}

The study population included 519 men with a mean age of 74.0 years (standard deviation, 5.8) and 164 (31.6\%) with sarcopenia. Severe ED was present in 272 men (52.4\%) at baseline. Compared with the non-sarcopenic men, those 
Table 2. Association of sarcopenia and its components with prevalent erectile dysfunction at baseline

\begin{tabular}{|c|c|c|c|c|}
\hline \multirow{2}{*}{ Variable } & \multirow{2}{*}{ No. with ED (\%) } & \multicolumn{3}{|c|}{ Odds ratio ( $95 \%$ confidence interval) } \\
\hline & & Model 1 & Model 2 & Model $_{3}$ \\
\hline Sarcopenia & 272 & & & \\
\hline Absent & $152(42.8)$ & Reference & Reference & Reference \\
\hline Present & $120(73.2)$ & $3.64(2.45-5.50)$ & $2.01(1.29-3.16)$ & $1.89(1.18-3.03)$ \\
\hline$p$ value & $<0.001$ & $<0.001$ & 0.002 & 0.008 \\
\hline \multicolumn{5}{|c|}{ Sarcopenia measures } \\
\hline \multicolumn{5}{|c|}{ Decreased muscle mass } \\
\hline Absent & & Reference & Reference & Reference \\
\hline Present & & $2.39(1.58-3.60)$ & $1.68(1.08-2.61)$ & $1.63(1.03-2.60)$ \\
\hline$p$ value & & $<0.001$ & 0.022 & 0.039 \\
\hline \multicolumn{5}{|c|}{ Slow gait speed } \\
\hline Absent & & Reference & Reference & Reference \\
\hline Present & & $1.73(1.12-2.66)$ & $1.21(0.76-1.93)$ & $1.04(0.65-1.68)$ \\
\hline$p$ value & & 0.013 & 0.419 & 0.865 \\
\hline \multicolumn{5}{|c|}{ Decreased grip strength } \\
\hline Absent & & Reference & Reference & Reference \\
\hline Present & & $2.43(1.56-3.80)$ & $1.59(0.98-2.58)$ & $1.51(0.92-2.48)$ \\
\hline$p$ value & & $<0.001$ & 0.063 & 0.107 \\
\hline
\end{tabular}

Model 1, unadjusted in the analysis of sarcopenia. Adjusted for decreased muscle mass, slow gait speed, and decreased grip strength in the analysis of sarcopenia components; Model 2, model 1 plus age; Model 3, model 2 plus smoking, cardiovascular disease, diabetes mellitus, depression, and polypharmacy.

$\mathrm{ED}$, erectile dysfunction.

with sarcopenia were older (mean age, 77.3 years vs. 72.4 years; $p<0.001$ ) and had a poorer health status based on a higher prevalence of cognitive dysfunction $35.4 \%$ vs. $7.9 \%, p<0.001)$, higher risk of malnutrition $(56.1 \%$ vs. $20.6 \%, p<0.001$ ), ADL disability (14.6\% vs. $6.5 \%, p<0.003$ ), and IADL disability (25.6\% vs. $9.9 \%)$. Other comorbid conditions were not statistically different (Table 1).

\section{Association of sarcopenia and each sarcopenia com- ponent with prevalent erectile dysfunction}

The prevalence of severe ED at baseline was higher in sarcopenic men (73.2\% vs. $42.8 \%$; OR, 3.64; $95 \%$ CI, 2.45 to 5.50; $p<0.001$ ) (Table 2). The association was attenuated with adjustment for age (OR, 2.01; 95\% CI, 1.29 to 3.16; $p=0.002$ ) and additionally for smoking, CVD, diabetes mellitus, depression, and polypharmacy (OR, 1.89; 95\% CI, 1.18 to $3.03 ; p=0.008$ ).

Each sarcopenia component was associated with severe ED after adjusting the other components: the ORs were 2.39 (95\% CI, 1.58 to $3.60 ; p<0.001$ ) for decreased muscle mass, 1.73 (95\% CI, 1.12 to $2.66 ; p=0.013$ ) for slow gait speed, and 2.43 (95\% CI, 1.56 to 3.80; $p<0.001$ ) for decreased grip strength (Table 2). The associations were attenuated with adjustment for age and additional risk factors for ED. The association of decreased muscle mass with severe ED remained statistically significant (OR, 1.63; 95\% CI, 1.03 to 2.60; $p=0.039$ ). The OR for decreased grip strength was similar, but not statistically significant (OR, 1.51; 95\% CI, 0.92 to $2.48 ; p=0.107$ ).

\section{Association of sarcopenia and each sarcopenia com- ponent with incident erectile dysfunction}

Out of 197 patients, the 1-year incidence of severe ED was $26.4 \%(n=52)$. Among the 52 patients with severe ED, 22.7\% ( $n=37)$ were without sarcopenia and $44.1 \%(n=15)$ had sarcopenia (OR, 2.69; 95\% CI, 1.25 to 5.81; $p=0.012$ ). However, the association between sarcopenia and incident severe ED was attenuated with adjustment for patient age (OR, 1.70; $95 \% \mathrm{CI}, 0.73$ to $3.95 ; p=0.215)$ and other risk factors (OR, 1.87; 95\% CI, 0.79 to $4.43 ; p=0.155$ ) (Table 3 ). 
Table 3. Association of sarcopenia and its components with incident erectile dysfunction at 1 year

\begin{tabular}{|c|c|c|c|c|}
\hline \multirow{2}{*}{ Variable } & \multirow{2}{*}{ No. with ED (\%) } & \multicolumn{3}{|c|}{ Odds ratio ( $95 \%$ confidence interval) } \\
\hline & & Model 1 & Model 2 & Model $_{3}$ \\
\hline Sarcopenia & 52 & & & \\
\hline Absent & $37(22.7)$ & Reference & Reference & Reference \\
\hline Present & $15(44.1)$ & $2.69(1.25-5.81)$ & $1.70(0.73-3.95)$ & $1.87(0.79-4.43)$ \\
\hline$p$ value & 0.01 & 0.012 & 0.215 & 0.155 \\
\hline \multicolumn{5}{|c|}{ Sarcopenia Measures } \\
\hline \multicolumn{5}{|c|}{ Decreased muscle mass } \\
\hline Absent & & Reference & Reference & Reference \\
\hline Present & & $3.02(1.43-6.35)$ & $2.17(0.98-4.77)$ & $2.54(1.11-5.81)$ \\
\hline$p$ value & & 0.004 & 0.055 & 0.027 \\
\hline \multicolumn{5}{|c|}{ Slow gait speed } \\
\hline Absent & & Reference & Reference & Reference \\
\hline Present & & $2.84(1.26-6.39)$ & $2.49(1.08-5.76)$ & $2.80(1.18-6.62)$ \\
\hline$p$ value & & 0.012 & 0.033 & 0.019 \\
\hline \multicolumn{5}{|c|}{ Decreased grip strength } \\
\hline Absent & & Reference & Reference & Reference \\
\hline Present & & $1.14(0.49-2.65)$ & $0.83(0.34-2.04)$ & $0.76(0.30-1.91)$ \\
\hline$p$ value & & 0.763 & 0.689 & 0.564 \\
\hline
\end{tabular}

Model 1, unadjusted in the analysis of sarcopenia. Adjusted for decreased muscle mass, slow gait speed, and decreased grip strength in the analysis of sarcopenia components; Model 2, model 1 plus age; Model 3, model 2 plus smoking, cardiovascular disease, diabetes mellitus, depression, and polypharmacy.

$\mathrm{ED}$, erectile dysfunction.

According to each sarcopenia component, the incidence of severe ED was higher in men with decreased muscle mass (45.2\% vs. $21.3 \%$; OR, 3.02; $95 \%$ CI, 1.43 to $6.35 ; p=$ 0.004 ) and slow gait speed (32.1\% vs. 14.3\%; OR, 2.84; $95 \%$ CI, 1.26 to $6.39 ; p=0.012$ ) compared to those with normal muscle mass and normal gait speed (Fig. 2). Therefore, the difference in the incidence of severe ED over weak grip strength was not significant (32.4\% vs. $25.2 \%$; OR, 1.14; $95 \%$ CI, 0.49 to $2.65 ; p=0.763$ ). Adjustment for age and additional risk factors moderately reduced the associations, but decreased muscle mass (OR, 2.54; 95\% CI, 1.11 to $5.81 ; p=0.027)$ and slow gait speed (OR, 2.80; 95\% CI, 1.18 to $6.62 ; p=0.019$ ) remained significantly associated with severe ED. After adjustment, grip strength was not significantly associated with the incidence of severe ED (OR, 0.76 ; $95 \%$ CI, 0.30 to $1.91 ; p=0.564$ ).

\section{DISCUSSION}

In a population-based study of community-dwelling, older Korean men, we found that the prevalence of severe ED and sarcopenia was $52.4 \%$ and $31.6 \%$, respectively. Our cross-sectional analysis showed that those men with sarcopenia were more likely to have severe ED than those without sarcopenia. Although the association between sarcopenia and severe ED 1 year after the study began did not reach statistical significance and decreased muscle mass and slow gait speed were independently associated with severe ED.

While the frequency of sexual activity decreases with aging, ED is the most common sexual problem in men $[1,30]$. Only a few studies examined the characteristics of older adults with ED. Those studies used a sexual activity questionnaire or a 15-question version of IIEF to measure ED in mainly urban area dwelling men and reported the prevalence of severe ED ranging from $15.0 \%$ 


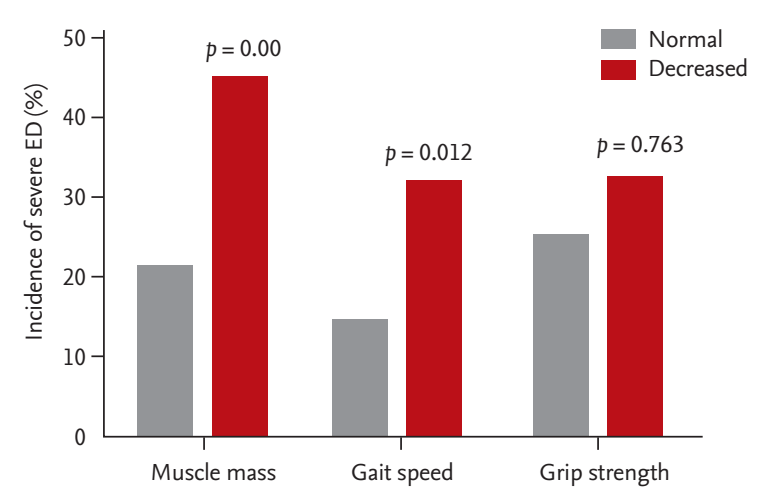

Figure 2. Incidence of severe ED according to each sarcopenia component. Decreased muscle mass; the sex-specific lowest quintile of ASM/height ${ }^{2}$. Slow gait speed; gait speed $<0.8 \mathrm{~m} / \mathrm{sec}$. Decreased grip strength; grip strength $<26 \mathrm{~kg}$. ED, erectile dysfunction; ASM, appendicular skeletal mass.

to $49.4 \%$ [31-33], and which was lower than that in our study. The use of different ED questionnaires or the differences in health status of the study populations may explain the difference in the prevalence of severe ED.

The physiology of penile erection involves relaxation of the penile smooth muscle, enlarged sinusoids, and substantial venous plexus compression. Any situation that can interrupt such mechanisms can cause ED. Impaired parasympathetic outflow or increased sympathetic outflow can inhibit relaxation forces. In addition to patient age, diabetes mellitus, dyslipidemia, metabolic syndrome, atherosclerosis, and smoking can directly reduce the activity of nitric oxide synthase and induce apoptosis of endothelial and smooth muscle cells. And although the mechanism has not yet been fully elucidated, low androgen hormone level and patient depression are also known to affect ED [1].

Sarcopenia is caused primarily by age-related changes, such as sex hormones, apoptosis, and mitochondrial dysfunction. Secondarily, endocrine disorder, e.g., corticosteroid, insulin resistance, abnormal thyroid function, growth hormone, and insulin-like growth factor 1, neuro-degenerative disease, inadequate nutrition, and disuse are known to cause sarcopenia [8,25]. These risk factors demonstrate the overlap of sarcopenia and ED $[8,25]$. In particular, low levels of androgen are associated with decreased anabolism of skeletal muscle and can consequently cause decreased muscle mass and strength or sarcopenia. In addition, supplementation of the androgen hormone has been cautiously suggested in the treatment of sarcopenia $[13,14]$. In a meta-analysis, androgen replacement showed increases in overall muscle strength [34]. Sharing of these risk factors and the association of androgen hormone may explain the association between sarcopenia and ED. Previously, the association between ED and sarcopenia in men with diabetes has been reported in a study [15], although the results might not be generalizable to general, community-dwelling, older adults who have and do not have diabetes. Therefore, to our knowledge, our study is the first to report that sarcopenia may be a risk factor for severe ED, independently of established risk factors for ED.

Our results have several potential clinical implications. Recently, the association between ED and frailty, which shows the general performance and physiologic reservoir of older adults, has been suggested/reported [19]. Among sarcopenic older men, unidentified ED patients can be treated to improve their quality of life, and it can be a sentinel check-point to reveal unrecognized frailty and cardiovascular risk factors. In addition, a person with decreased muscle mass and slow gait speed might be able to receive education regarding the future severe ED risk and pre-emptively intervene in order to avoid severe ED in the future.

There are strengths and limitations to our study. Because ASPRA has a high participation rate of eligible individuals, selection bias due to non-participation is unlikely [16]. We administered standardized assessments, including a IIEF-5 questionnaire, comprehensive geriatric assessment, and bioelectrical impedance analysis [18,35], with high completion rates. Among 519 participants at the baseline, 407 men participated in the 1-year follow-up and among the 247 men without severe ED at baseline, 197 were reassessed for ED 1 year later. However, the baseline characteristics between those with IIEF-5 data (407 men) and without (112 men) at 1 year were similar (Supplementary Table 1). Because this study was a population-based study targeted to the community-dwelling, older adults, we provided the clinical evidence to explain the relationship between sarcopenia and erectile dysfunction, but could not show the laboratory findings regarding cellular changes or hormonal levels that further support our report. Subsequent studies are likely to be required and to be designed considering the linking mechanism between sarcopenia and erectile dysfunction. 
Our study was underpowered to detect a clinically meaningful increase in the risk of severe ED over 1 year, and which resulted in a wide $95 \%$ CI. Nonetheless, two of the three components of sarcopenia, i.e., decreased muscle strength and slow gait speed, were associated with the incidence of severe ED at 1 year, and which suggests sarcopenia as a risk factor for future severe ED. Additional follow-up data from the ASPRA cohort will allow more precise estimation of the association. Lastly, our results from older Korean men who live in rural communities may not generalize to older men who live in urban areas or other countries. Our results should be interpreted with caution until further validation in other populations.

In conclusion, sarcopenia was associated with severe ED, and components of sarcopenia-slow gait speed and decreased muscle mass were independently associated with the incidence of severe ED at 1 year in community-dwelling, older men. Further research is warranted to examine whether an intervention targeting these components can prevent severe ED.

\section{KEY MESSAGE}

1. The prevalence of severe erectile dysfunction was high among older Korean men.

2. Sarcopenia was significantly associated with severe erectile dysfunction at baseline.

3. Two sarcopenia components, i.e., decreased muscle mass and slow gait speed, were associated with the incidence of severe erectile dysfunction at 1 year.

\section{Conflict of interest}

Dae Hyun Kim is a consultant to Alosa Health, a nonprofit educational organization with no relationship to any drug or device manufacturers.

\section{Acknowledgments}

We are indebted to public health professionals and nurses of Pyeongchang County Hospital, Public Health Center, and Community Health Posts for their administrative support and efforts in patient enrollment and measurements. Otherwise, sponsors did not have any role in the study design, analysis and interpretation of data, in the writing of the article, and in the decision to submit the article for publication. The Aging Study of Pyeongchang Rural Area Intervention Study was supported by Pyeongchang County Hospital, Pyeongchang County, Gangwon Province, Korea. This study was also supported in part by Paul Park from Asan Institute for Life Sciences and Corporate Relations of Asan Medical Center, Seoul, Korea. Dr. Dae Hyun Kim is supported by grants Ro1AGo56368, P3oAG031679, and $\mathrm{P}_{30} \mathrm{AGo} 48785$ from the National Institute on Aging.

\section{REFERENCES}

1. McVary KT. Clinical practice. Erectile dysfunction. N Engl J Med 2007;357:2472-2481.

2. Ayta IA, McKinlay JB, Krane RJ. The likely worldwide increase in erectile dysfunction between 1995 and 2025 and some possible policy consequences. BJU Int 1999;84:50-56.

3. Rosen RC, Wing R, Schneider S, Gendrano N 3rd. Epidemiology of erectile dysfunction: the role of medical comorbidities and lifestyle factors. Urol Clin North Am 2005;32:403-417.

4. Johannes CB, Araujo AB, Feldman HA, Derby CA, Kleinman KP, McKinlay JB. Incidence of erectile dysfunction in men 40 to 69 years old: longitudinal results from the Massachusetts male aging study. J Urol 2000;163:460-463.

5. Malavige LS, Jayaratne SD, Kathriarachchi ST, Sivayogan S, Ranasinghe P, Levy JC. Erectile dysfunction is a strong predictor of poor quality of life in men with type 2 diabetes mellitus. Diabet Med 2014;31:699-706.

6. Delmonico MJ, Harris TB, Visser M, et al. Longitudinal study of muscle strength, quality, and adipose tissue infiltration. Am J Clin Nutr 2009;90:1579-1585.

7. Beaudart C, Biver E, Reginster JY, et al. Validation of the SarQoL ${ }^{\circledR}$, a specific health-related quality of life questionnaire for Sarcopenia. J Cachexia Sarcopenia Muscle 2017;8:238-244.

8. Chen LK, Liu LK, Woo J, et al. Sarcopenia in Asia: consensus report of the Asian Working Group for Sarcopenia. J Am Med Dir Assoc 2014;15:95-101.

9. Cruz-Jentoft AJ, Bahat G, Bauer J, et al. Sarcopenia: revised European consensus on definition and diagnosis. Age Ageing 2019;48:16-31.

10. Seftel AD. Erectile dysfunction in the elderly: epidemiology, etiology and approaches to treatment. J Urol 
2003;169:1999-2007.

11. Bhasin S, Cunningham GR, Hayes FJ, et al. Testosterone therapy in adult men with androgen deficiency syndromes: an endocrine society clinical practice guideline. J Clin Endocrinol Metab 2006;91:1995-2010.

12. Rajfer J. Relationship between testosterone and erectile dysfunction. Rev Urol 2000;2:122-128.

13. Basualto-Alarcon C, Varela D, Duran J, Maass R, Estrada M. Sarcopenia and androgens: a link between pathology and treatment. Front Endocrinol (Lausanne) 2014;5:217.

14. Shin MJ, Jeon YK, Kim IJ. Testosterone and sarcopenia. World J Mens Health 2018;36:192-198.

15. Ucak S, Sivritepe R, Kara O, et al. Association between sarcopenia and erectile dysfunction in males with type II diabetes mellitus. Aging Male 2019;22:20-27.

16. Jung HW, Jang IY, Lee YS, et al. Prevalence of frailty and aging-related health conditions in older Koreans in rural communities: a cross-sectional analysis of the aging study of Pyeongchang rural area. J Korean Med Sci 2016;31:345-352.

17. Jang IY, Jung HW, Lee CK, Lee YS, Lee E, Kim DH. Comparison between Korean version of physical activity scale for the elderly and international physical activity questionnaire-short form in evaluation of frailty phenotype. Ann Geriatr Med Res 2017;21:101-107.

18. Rosen RC, Cappelleri JC, Smith MD, Lipsky J, Pena BM. Development and evaluation of an abridged, 5-item version of the International Index of Erectile Function (IIEF5) as a diagnostic tool for erectile dysfunction. Int J Impot Res 1999;11:319-326.

19. Jang IY, Lee CK, Jung HW, et al. Urologic symptoms and burden of frailty and geriatric conditions in older men: the Aging Study of PyeongChang Rural Area. Clin Interv Aging 2018;13:297-304.

20. Kim M, Shinkai S, Murayama H, Mori S. Comparison of segmental multifrequency bioelectrical impedance analysis with dual-energy X-ray absorptiometry for the assessment of body composition in a community-dwelling older population. Geriatr Gerontol Int 2015;15:1013-1022.

21. Hachiya M, Murata S, Otao H, Kamijou K, Mizota K, Asa$\mathrm{mi}$ T. Reproducibility and validity of the 50-meter walking test in community-dwelling elderly. J Phys Ther Sci 2015;27:1511-1514.

22. Mathiowetz V, Rennells C, Donahoe L. Effect of elbow position on grip and key pinch strength. J Hand Surg Am 1985;10:694-697.

23. Peters DM, Fritz SL, Krotish DE. Assessing the reliabili- ty and validity of a shorter walk test compared with the 10-meter walk test for measurements of gait speed in healthy, older adults. J Geriatr Phys Ther 2013;36:24-30.

24. Jang HC. How to diagnose sarcopenia in Korean older adults? Ann Geriatr Med Res 2018;22:73-79.

25. Cruz-Jentoft AJ, Baeyens JP, Bauer JM, et al. Sarcopenia: European consensus on definition and diagnosis. Report of the European Working Group on Sarcopenia in Older People. Age Ageing 2010;39:412-423.

26. Won CW, Yang KY, Rho YG, et al. The development of Korean Activities of Daily Living (K-ADL) and Korean Instrumental Activities of Daily Living (K-IADL) scale. J Korean Geriatr Soc 2002;6:107-120.

27. Kang Y, Na DL, Hahn S. A validity study on the Korean Mini-Mental State Examination (K-MMSE) in dementia patients. J Korean Neurol Assoc 1997;15:300-308.

28. Radloff LS. The CES-D scale: a self-report depression scale for research in the general population. Appl Psychol Meas 1977;1:385-401.

29. Rubenstein LZ, Harker JO, Salva A, Guigoz Y, Vellas B. Screening for undernutrition in geriatric practice: developing the short-form mini-nutritional assessment (MNASF). J Gerontol A Biol Sci Med Sci 2001;56:M366-M372.

30. Lindau ST, Schumm LP, Laumann EO, Levinson W, O'Muircheartaigh CA, Waite LJ. A study of sexuality and health among older adults in the United States. N Engl J Med 2007:357:762-774.

31. Marumo K, Nakashima J, Murai M. Age-related prevalence of erectile dysfunction in Japan: assessment by the International Index of Erectile Function. Int J Urol 2001;8:53-59.

32. Feldman HA, Goldstein I, Hatzichristou DG, Krane RJ, McKinlay JB. Impotence and its medical and psychosocial correlates: results of the Massachusetts Male Aging Study. J Urol 1994;151:54-61.

33. Hyde Z, Flicker L, Hankey GJ, et al. Prevalence and predictors of sexual problems in men aged 75-95 years: a population-based study. J Sex Med 2012;9:442-453.

34. Ottenbacher KJ, Ottenbacher ME, Ottenbacher AJ, Acha AA, Ostir GV. Androgen treatment and muscle strength in elderly men: a meta-analysis. J Am Geriatr Soc 2006;54:16661673 .

35. Jung HW, Yoo HJ, Park SY, et al. The Korean version of the FRAIL scale: clinical feasibility and validity of assessing the frailty status of Korean elderly. Korean J Intern Med 2016;31:594-600. 
Supplementary Table 1. Baseline characteristics between those with IIEF-5 data and without at 1 year

\begin{tabular}{lccc}
\hline Characteristic & With IIEF-5 $(\mathrm{n}=407)$ & Without IIEF-5 $(\mathrm{n}=112)$ & $p$ value \\
\hline Age, yr & $73.8 \pm 5.29$ & $74.5 \pm 7.27$ & 0.267 \\
Smoking & $61(15.0)$ & $26(23.2)$ & 0.046 \\
CVD & $240(59.0)$ & $65(58.0)$ & 0.914 \\
Diabetes & $70(17.2)$ & $26(23.2)$ & 0.169 \\
Depression & $7(1.7)$ & $5(4.5)$ & 0.145 \\
Polypharmacy & $73(17.9)$ & $26(23.2)$ & 0.222 \\
Sarcopenia & $125(30.7)$ & $39(34.8)$ & 0.423 \\
\hline
\end{tabular}

Values are presented as mean \pm SD or number (\%).

IIEF-5, the 5-item International Index of Erectile Function questionnaire; CVD, cardiovascular disease. 